\title{
Effect of Inspiratory Muscle Training versus Breathing Exercise Training to Enhance the Sprint Performance and PiMax on Wheelchair Athletes with Spinal Cord Injury
}

\section{Piyush Singh*}

Indian Spinal Injuries Centre, Institute of Rehabilitation Sciences, Vasant Kunj, New Delhi, India

"Corresponding author: Piyush Singh: Assistant Professor, Indian Spinal Injuries Centre, Institute of Rehabilitation Sciences, Vasant Kunj, New Delhi, India, Tel: 09971009811; E-mail: piyushphysio@gmail.com

Rec date: Mar 24, 2016; Acc date: Jun 17, 2016; Pub date: Jun 20, 2016

Copyright: (c) 2016 Singh P, et al. This is an open-access article distributed under the terms of the Creative Commons Attribution License, which permits unrestricted use, distribution, and reproduction in any medium, provided the original author and source are credited.

\begin{abstract}
Background and purpose: Spinal cord injury can be one of the most fatal experience for the individuals who sustains it, as it not only endows one with devastating effects of disability but as well renders significant effects on personal, social as well as economic aspects of a person's life. Wheelchair basketball is a sport which requires efficient respiratory capacity or function to sustain in the game for prolonged period of time. In higher thoracic Spinal Cord Injury, respiratory function reduces and due to fatigue patient becomes dyspneic easily even after small workout.
\end{abstract}

Objective: To evaluate the effectiveness of Inspiratory muscle trainer (power breathe) and breathing exercise on sprint timing and maximum inspiratory capacity or pressure inside the lungs.

Methodology: Samples of 45 subjects with upper thoracic (T1-T6 level) wheelchair basketball athletes was recruited and were randomly divided into three groups: IMT, breathing exercise and control. Baseline scores of PiMax and sprint time were assessed using inspiratory monometer apparatus and $20 \mathrm{~m}$ shuttle test respectively. Six weeks training was given to both the experimental groups and post intervention scores were measured after every 2 weeks.

Results: Highly significant differences in IMT and breathing exercise groups were observed $(p=0.005)$. However, at last week of training improvement was not so significant in IMT group. Significant differences were found within and between groups.

Conclusion: Our data indicates that IMT and Breathing exercise have a significant effect on PiMax, sprint and dyspnea in recreational wheelchair basketball players by assessing through Borg scale of perceived exertion.

Keywords: IMT: Inspiratory Muscle Trainer; PiMax: Inspiratory maximum pressure; Breathing exercise; Sprint timing

\section{Introduction}

American Spinal Cord Injury Association defines spinal cord injury as an insult to the spinal cord resulting in a change, either temporary or permanent, in its normal motor, sensory or autonomic function' [1].

Most commonly affected age group with spinal cord injury is between 20-29 years which accounts for $34.18 \%$ of injured group and remaining $68.54 \%$ fall under the age of 40 years. Mean age group for injury remains at 34.4 years [2].

Spinal cord injury causes paralysis of upper and lower extremity and can lead to chronic respiratory insufficiency due to paralysis of respiratory muscles which are innervated below the level of lesion [3].

Injury to cervical spine causes major respiratory complications such as micro atelectasis, pneumonia and respiratory failure resulting from decreased lung and chest wall compliance. Ventilatory functions remain less affected in patients with lower thoracic and lumbar lesions [4-7].
Spinal cord injury gives rise to different types of dysfunctions such as impaired inspiratory capacity, reduced respiratory muscle strength, fatigue and increased effort of breathing due to paradoxical chest wall movement. These complications lead to increased production of secretions and reduced cough effectiveness [4-6,8].

Most common complication in T1-T12 injury group is atelectasis found in $65 \%$ of population. Summarily pneumonia, atelectasis and ventilatory failure are the commonest respiratory complication affecting most of the spinal cord injury groups $[4,9,10]$.

During normal respiratory biomechanics the diaphragm contraction can elevate the rib cage and increases intra-abdominal pressure. In patients with spinal cord injury, the abdomen becomes highly compliant, reducing the effectiveness of diaphragm action on rib cage. This can lead to smaller-than-normal increase in intraabdominal pressure during inhalation [11].

Patients suffering with tetraplegia and high paraplegia suffer with mucus retention and atelectasis due to ineffective cough, as expiratory muscle function is more compromised than the inspiratory [12]. 
In traumatic tetraplegics reduced tidal volume reduces and increased abdominal compliance, contributes in reducing rib cage expansion. This phenomenon normally happens because of sternocleidomastoid contraction which results in pure "pump-handle" action and thus reducing abdominal diameter and transverse diameter of thorax [13,14].

Since early 1980s inspiratory muscle training (IMT) is used in literatures. IMT has been proven effective in conditions like chronic obstructive pulmonary disease, neuromuscular disorders, asthma and chronic heart failure. It has positive effect on exercise capacity and health related quality of life by improving inspiratory muscle strength and endurance [15]. There are three different types of inspiratory muscle trainer including resistance trainers, normocapnic hyperventilation trainers and threshold trainers [15].

High level of evidence literature has supported use of IMT in adults with stable Chronic Obstructive pulmonary disease (COPD) by targeted inspiratory resistive or threshold IMT [16].

IMT significantly improves exercise capacity by increasing inspiratory muscle function through fixed load and full inspiratory volume IMT [17].

As per Leith and Bradley normal individuals can improve their ventilatory muscle strength and endurance with specific training program of repeated static maximal inspiratory and expiratory maneuvers against obstructed airways [18].

\section{Methods}

Ethical clearance was obtained from the Institutional Ethical committee of Indian Spinal Injuries Centre. A sample of 45 individuals diagnosed with spinal cord injury (AIS-A and AIS-B) participated in the study. Few subjects were selected from Indian Spinal Injuries Centre hospital and few others from Research and Referral Army hospital, New Delhi.

Individuals were recruited in the study based on following inclusion criteria: Individuals diagnosed with traumatic SCI of AIS-A and AIS$B$, minimum of one year of span in injury. Subjects were excluded from the study on the following basis: subjects with pre-existing lower limb pathology, deformity or musculoskeletal problem or any joint pain and any other neurological disorder and health condition that preclude participation like uncontrolled hypertension, COPD, osteoporosis or cardiac problems, peripheral neuropathy and subjects diagnosed with any systemic illness.

A pre and post-test between group study designs was used. Participants were then explained about the procedure of the study and written consent was obtained from each participant. Participants were randomly divided into two groups i.e., Group 1 (Inspiratory muscle training) and Group 2 (Breathing training). Participants were tested for sprint timing through Shuttle test and inspiratory maximum pressure via inspiratory monometer apparatus; Borg perceived exertion scale is used for measuring exertion level. All the measurements were followed after every two weeks during protocol completion.

Participants in Group 1 received Inspiratory muscle training (IMT) for six weeks two times a day. Each session lasted for approximately 5 min and warm up activity was taught prior to sprinting. Post results were obtained after every two weeks.

Participants in Group 2 received breathing training exercise program for six weeks three times in a day. Each session lasted for approximately for 10 minutes. At the end of six weeks, participants were assessed for sprint performance and PiMax values.

\section{Data Analysis}

The SPSS 21.0 statistical package (SPSS Inc., Chicago IL, USA) was used for all the statistical analysis. Means and standard deviations were compared for all variables. A two-way analysis of variance with repeated measurements was used with as within-subject variable training (pre and post) and as between subject variable group (IMT vs. BT). Significance was assumed at $\mathrm{P} \leq 0.05$. A Bonferroni post hoc test was applied to determine the location of any significant main effects.

\section{Results}

In the present study a total of 30 wheelchair recreational players with high thoracic spinal cord injury were recruited. The study consisted of majority in male participants and few female due to unavailability. The mean and SD of age (year) of the inspiratory muscle training group was $40.50 \pm 9.70$.

Between group analysis for pre intervention values for experimental group 1 (IMT) and experimental group 2 (BT) was done using independent T-test and Sprint timing was measured. Pre intervention data analysis for sprint and PiMax showed no significant difference.

The data analysis for post intervention values of experimental group 1 (IMT) and group 2 (BT) was also done using independent T-test and PiMax value was measured, showed significant changes at the end of 6 weeks.

Sprint Result of Group 1 and 2: Pre intervention score analysis of experimental Group 1 and Group 2 was $17.286 \pm 1.3513$ and $17.844 \pm$ 1.239 respectively (Figure 1 ).

After 6 weeks of intervention sprint mean and SD for group 1 and 2 was $18.53 \pm 7.21$ and $15.89 \pm 1.435$ respectively.

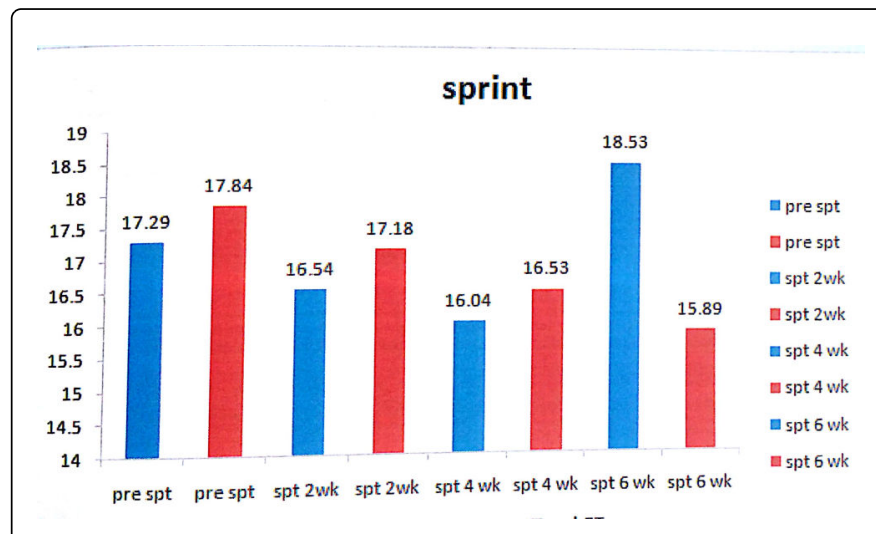

Figure 1: Intervention sprint mean and SD for groups 1 and 2.

PiMax result of Groups 1 and 2: Pre intervention PiMax mean and SD of groups 1 and 2 was $36.007 \pm 1.795$ and $35.356 \pm 2.551$ respectively (Figure 2).

After 6 weeks of intervention score analysis of PiMax mean and SD for groups 1 and 2 was $39.48 \pm 1.879$ and $38.54 \pm 2.036$ respectively. 


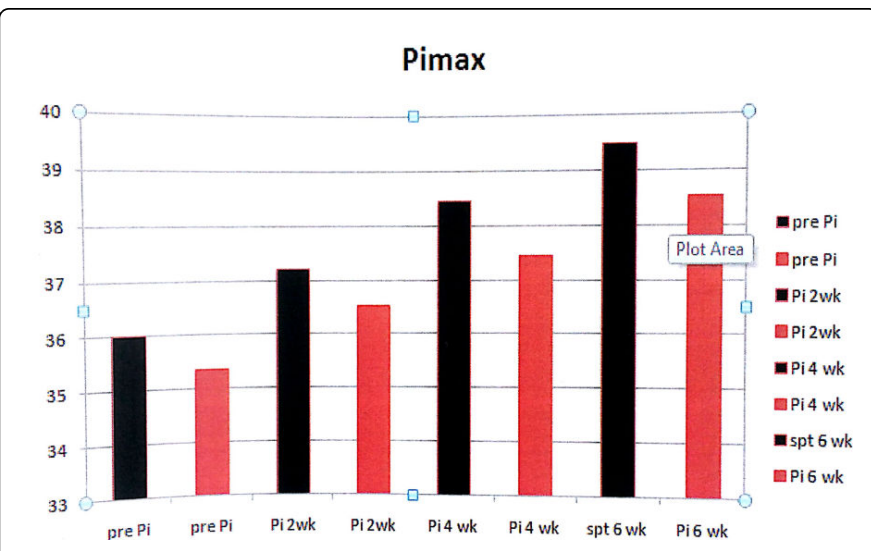

Figure 2: Intervention PiMax mean and SD of groups 1 and 2.

\section{Discussion}

This study deals with the effect of inspiratory muscle training versus breathing training on sprint performance and PiMax on higher thoracic spinal cord injury patients, participants informed consent was obtained before data collection, thirty recreational basketball players participated in the study. Disabilities of the participants included only SCI, with minimum time of disability to one year.

No study has previously shown the effect of IMT on sprint performance and PiMax in higher thoracic spinal cord injury patients, although there are studies which show the effect of these two interventions on cyclists, swimmers and COPD patients with the basic aim to improve the aerobic endurance and respiratory function [9,19-21]. There is an existence of evidence documenting the effect of IMT on SCI patients with decrease in dyspnoea and improved strength and endurance [22].

The high thoracic spinal cord injury patients were recruited of ASIA-A and ASIA-B, as per international standard for neurological classification of spinal cord injury revised 201, ASIA impairment scale [2]. Injury to high thoracic spinal cord leads to highly compromised respiratory system due to the affection of respiratory muscles [22]. Higher lesions result in greater denervation of expiratory and inspiratory muscles thus lesions of cervical and upper thoracic regions results in greater respiratory distress [23]. As seen in able bodied individuals evidence suggest use of exercise methods to improve cardiovascular health in Spinal cord injury patients. It is important to use exercise methods as physical inactivity in SCI patients can lead to secondary conditions such as cardiovascular disease, diabetes, osteoporosis and obesity [23].

Normal functioning of respiration involves muscles of chest, back and abdominal area. Dyspnoea during sprinting among wheelchair recreational basketball players can be manage with the good respiratory function [22]. Lack of sympathetic innervations of the heart there is a loss of sympathetic neural control which can be accounted for the lowest $\mathrm{Vo} 2 \mathrm{Pk}$ recorded in above T1-T6 level of injury [6].

A simple test of evaluating maximum inspiratory pressure can indicate the amount of muscle weakness and can also be used as a repetitive test to follow the course of disease. Maximum inspiratory pressure can be in normal ranges in patients with some muscular disease but they develop abnormally rapid fatigue on exercise or during sprinting [24].

The primary findings of this study indicates significant effect of IMT on sprint performance and PiMax but during sixth week the effect remained static as the majority of participants develop maximum inspiratory pressure and training with IMT on higher level (4/5) made them to finish the training early before reaching the level 9 . There was an adaptation to the equipment resistance.

This indicates that even minimal intensity IMT is sufficient to improve respiratory muscle function because IMT predominantly concerns inspiration; most previous studies have focussed on maximum inspiratory pressure rather than maximum expiratory pressure. Grandas et al. noted that dyspnea in chronic SCI patients may be because of impaired ventilatory muscle function and the adaptive strategies are used to alleviate the problem [9].

Similar significant improvements in the sprint and PiMax were seen in breathing training group. This conclude that pulmonary rehabilitation exercise protocol prescribed to the SCI patients with reduced pulmonary function is very much effective to provide a positive outcome. Pulmonary exercises were simple and effective therapy without any need for purchase of any sophisticated instrument.

Only when such respiratory muscle training is sustained in a prolonged way it will help inducing changes that may protect against both the development of respiratory muscle fatigue and recurrent respiratory infection. This one of the important factor for wheelchair basketball players as they need an efficient respiratory functional endurance so that they can compete and sustain in the sports for prolonged period of time.

A study conducted in All India Institute of Medical Sciences, New Delhi on efficacy of breathing training to improve the pulmonary functioning tetraplegic and high paraplegic subjects. In this study high paraplegic individuals demonstrated significant improvement in FVC, FEV and maximum inspiratory pressure after six weeks of exercise programme [25].

Our data indicates that both Inspiratory muscle training and breathing training are equally effective in improving sprint timing and PiMax or to enhance the respiratory function in those who had SCI of higher thoracic and might help to decrease the severity of recurring chest infection, a common complication of people with spinal cord injury.

\section{Limitation of study}

Limitation of study is inclusion of only ASIA-A and ASIA-B. These two groups are separated from each other on neurological grounds and have hence differences among them. Inspiratory muscle trainer should be calibrated and absence of higher resistance level leads to stagnancy of improvement in athletes.

\section{Conclusion}

Our data indicates that both Inspiratory muscle trainer and breathing training have significant effect on PiMax $(\mathrm{p} \geq 0.001)$ and sprint $(p \geq 0.001)$ in wheelchair spinal cord injured paraplegic patients. Limitation in further improvement of IMT group is attributed to adaptation of subjects to certain last fixed level i.e., 9. 
Citation: $\quad$ Singh P (2016) Effect of Inspiratory Muscle Training versus Breathing Exercise Training to Enhance the Sprint Performance and PiMax on Wheelchair Athletes with Spinal Cord Injury. J Spine 5: 314. doi:10.4172/2165-7939.1000314

Page 4 of 4

\section{References}

1. Berlly M, Shem K (2007) Respiratory management during the first five days after spinal cord injury. J Spinal Cord Med 30: 309-318.

2. American Spinal Injury Association (2006) International standard for neurological classification of spinal cord injury. Revised Edition, Chicago: Respiratory Medicine 100: 1886-1895.

3. Bradley M, Leith DE (1976) Ventilatory muscle strength and Endurance training. J Appl Physiol 4: 508-516.

4. Brown R, DiMarco AF, Hoit JD, Garshick E (2006) Respiratory dysfunction and management in spinal cord injury. Respir Care 51: 853-868.

5. Chhabra HS, Arora M (2012) Demographic profile of traumatic spinal cord injuries admitted at Indian Spinal Injuries Centre with special emphasis on mode of injury: A retrospective study. Spinal Cord 50: 745-754.

6. Cooper A (1990) Wheelchair racing sports science: A review. J Rehabil Res Dev 27: 295-312.

7. Loveridge B, Badour M, Dubo H (1989) Ventilatory muscle endurance training in quadriplegia: Effects on breathing pattern. Paraplegia 27: 329-339.

8. Chiappa GR, Roseguini BT, Vieira PJ, Alves CN, Tavares A, et al. (2008) Inspiratory muscle training improves blood flow to resting and exercising limbs in patients with chronic heart failure. J Am Coll Cardiol 5: 0735-1097.

9. Crowe J, Lynne G, Brooks D, Reid D (2008) Inspiratory muscle training for individuals with chronic obstructive pulmonary diseases: A survey of Canadian physical therapists. J Physiother 5: 1708-8313.

10. David RH, Harris C (1987) Pulmonary complications of acute spinal cord injuries. Neurosurgery 21: 193-196.

11. Estenne M, De Troyer A (1986) The effects of tetraplegia on chest wall statics. Am Rev Respir Dis 134: 121-124.

12. Zupan A, Sarvin R, Erjavec T, Kralj A, Karcnik T, et al. (1997) Effects of respiratory muscle training and electrical stimulation of abdominal muscles on respiratory capabilities in tetraplegic. Spinal Cord 35: 540-545.

13. Fishburn MJ, Marino RJ, Ditunno JF Jr (1990) Atelectasis and pneumonia in acute spinal cord injury. Arch Phys Med Rehabil 71: 197-200.
14. Geddes LE, Reid DW, Crowe J, Brien K, Brooks D (2005) Inspiratory muscle training in adults with chronic obstructive pulmonary disease: A systematic review. Respir Med 99: 1440-1458.

15. Mansel JK, Norman JR (1990) Respiratory complications and management of spinal cord injuries. Chest 97: 1446-1452.

16. Hopman MT, van der Woude LH, Dallmeijer AJ, Snoek G, Folgering HT (1997) Respiratory muscle strength and endurance in individuals with tetraplegia. Spinal Cord 35: 104-108.

17. Liaw MY, Lin MC, Cheng PT, Wong MK, Tang FT (2000) Resistive inspiratory muscle training: its effectiveness in patients with acute complete cervical cord injury. Arch Phys Med Rehabil 81: 752-756.

18. Lisboa C, Muñoz V, Beroiza T, Leiva A, Cruz E (1994) Inspiratory muscle training in chronic airflow limitation: comparison of two different training loads with a threshold device. Eur Respir J 7: 1266-1274.

19. Lötters F, van Tol B, Kwakkel G, Gosselink R (2002) Effects of controlled inspiratory muscle training in patients with COPD: a meta-analysis. Eur Respir J 20: 570-576.

20. Hildegard SR, Montemayor Rubio T, Ortega Ruiz F, Cejudo Ramos P, Del Castillo Otero D (2001) Inspiratory muscle training in patients with COPD, Effect on dyspnea, exercise performance and quality of life. Chest 120: 748-756.

21. Hill K, Jenkins SC, Hillman DR, Eastwood PR (2004) Dyspnoea in COPD: can inspiratory muscle training help? Aust J Physiother 50: 169-180.

22. Goosey V, Foden E, Perret C, Dedgens H (2010) Effects of inspiratory muscle training on respiratory function and repetitive sprint performance in wheelchair basketball players. Br J Sports Med 44: 665-668.

23. Sheel AW, Reid WD, Townson AF, Ayas NT, Konnyu KJ (2008) (Spinal Cord Rehabilitation Evidence Research Team) Effects of exercise training and inspiratory muscle training in spinal cord injury: a systematic review. J Spinal Cord Med 31: 500-508.

24. Brooks D, O'Brien K, Geddes EL, Crowe J, Reid WD (2005) Is inspiratory muscle training effective for individuals with cervical spinal cord injury? A qualitative systematic review. Clin Rehabil 19: 237-246.

25. Suresh R (2004). A study of efficiency of breathing exercises to improve pulmonary function in tetraplegic and high paraplegic subjects. IJPMR $15,17-22$. 